\title{
Entropy
}

ISSN 1099-4300

(C) 2003 by MDPI

www.mdpi.org/entropy

\section{Extensive Generalization of Statistical Mechanics Based on Incomplete Information Theory}

\section{Qiuping A. Wang}

Institut Supérieur des Matériaux du Mans, 44, Avenue F.A. Bartholdi, 72000 Le Mans, France E-mail: awang@ismans.univ-lemans.fr

Received: 23 May 2002 / Accepted: 30 January 2003 / Published: 30 June 2003

\begin{abstract}
Statistical mechanics is generalized on the basis of an additive information theory for incomplete probability distributions. The incomplete normalization $\sum_{i=1}^{w} p_{i}^{q}=1$ is used to obtain generalized entropy $S=\sum_{i=1}^{w} p_{i}^{q} \ln p_{i}$. The concomitant incomplete statistical mechanics is applied to some physical systems in order to show the effect of the incompleteness of information. It is shown that this extensive generalized statistics can be useful for the correlated electron systems in weak coupling regime.
\end{abstract}

PACS numbers: 02.50.-r, 05.20.-y, 71.10.-w, 71.27.+a

Keywords: Statistical Mechanics, Generalized Statistics, Incomplete Information, Thermodynamics.

\section{Introduction}

If the coin of the Chevalier de Méré ${ }^{1}$ comes down heads for $49 \%$, tails must be $51 \%$ because it has only two sides. Since Pascal, this hypothesis has never been questioned.

Let us define an ensemble $\Omega$ of $N$ elements (e.g. number of tosses of a coin). Every element has $v$ possible states (sides of coin). A random variable (RV) of this ensemble is denoted by $\xi$ of which the value is $x_{i}$ at state $i$ with probability $p_{i}$. All observed values of $\xi$ constitute an ensemble $X=\left\{x_{1}, x_{2}, \ldots\right.$ 
,$\left.x_{v}\right\}$ with a probability distribution $P=\left\{p_{1}, p_{2}, . ., p_{v}\right\}$. If $v$ is the number of all the possible values of $\xi$, then $X$ is a complete ensemble and $\xi$ is called a complete random variables (CRV) [1]. In the toss of a coin, the coin position is a CRV having two values $(v=2)$ : heads or tails. $\xi$ is referred to as independent CRV if all its values are independent (e.g. the result of a toss has not any influence on the result of the next toss) and exclusive (e.g., heads can not be tails). In this case, $P$ is called a complete probability distribution (CPD) for which we have the following postulate :

$$
\sum_{i=1}^{v} p_{i}=1
$$

The corresponding mathematical framework for calculating $P$ is sometimes called Kolmogorov algebra of probability distribution [1]. Eq.(1) is the foundation of all probabilistic sciences using CPD. For the average value of certain quantity $\hat{O}$ of the ensemble $\Omega$, we should write

$$
\bar{O}=\sum_{i=1}^{v} p_{i} O_{i}
$$

where $O_{i}$ is the value of $\hat{O}$ in the state $i$.

It is noteworthy that the sum in these equations is over all possible states. Therefore, logically, all statistical theories constructed within Kolmogorov algebra of CPD should be applied to systems of which all the possible states are well-known and calculable so that we can count them to carry out the calculation of probability or of any quantity. In physics, this requires not only the exact Hamiltonian and solutions of the equation of motion, but also the mathematical tools allowing one to treat exactly and exhaustively the known states in phase space.

However, in Nature, there are systems about which we are incapable of treating all information. Many systems in physics theory are nothing but some isolated and simplified subsystems of the complex and messy world only partially known. The information needed to specify exactly these subsystems may not be completely accessible for us. In this case, strictly speaking, the CPD from which completely accessible information can be calculated may be invalid. If the inaccessible information is not negligible, we should address a more general distribution, that is the incomplete probability distributions (IPD) associated with the incomplete random variables of which we do not know all the possible values (i.e. $v$ may be greater or smaller than the real number of possible values). CPD is only a special case of IPD [1]. Very recently, a possible statistical theory for IPD was proposed [2-5]. This statistics based on the incomplete information consideration has been applied to a generalization of Boltzmann-Gibbs statistical mechanics (BGS), the so called nonextensive statistical mechanics (NSM) [6]. It is shown [3] that this nonextensive incomplete statistics (NIS) can indeed parallel the CPD version of NSM and avoid some theoretical difficulties. In addition, the incomplete nonextensive generalization of Fermi-Dirac distribution is proved to be the only generalized quantum distribution (among several ones) showing the same characteristics as the distribution of strongly correlated heavy fermions and so is possible to be applied to this kind of systems $[4,5]$.

In this paper, on the other hand, the idea of IPD will be used to generalize BGS for the cases where additive information and physical quantities hold. We will show that it is possible to use this extensive generalization of BGS to describe correlated electrons in the weak-coupling regime. 


\section{About Incomplete Distributions}

A "ideal" coin has only two sides. So we surely have $v=2$. But what about a coin having thick edge which possibly remains standing without being counted? And what is the value of $v$ for a quantum coin? a coin having overlapped heads and tails? For a coin having fractal or chaotic dynamics? These questions may appear comic. But many real systems can surely be regarded as one of these coins, especially the complex systems with correlation or effects of correlation that can not be exactly described with analytic methods. For this kind of systems, the equation of motion becomes incomplete in the sense either that some interactions are missing in the Hamiltonian or that its solution can not yield complete knowledge about the system. This incompleteness of knowledge makes it impossible to apply Kolmogorov theory because Eq.(1) and Eq.(2) can not be calculated. In this case, statistical theories for IPD are necessary.

The basic assumption of incomplete statistics [2] is that our knowledge about physical systems and the relevant probability distributions are incomplete, i.e. $\sum_{i=1}^{w} p_{i}=Q \neq 1$ [1] where $w$ is only the number of accessible states in phase space. So one can only write $\sum_{i=1}^{w} F\left(p_{i}\right)=1$ where $F$ is certain function of $p_{i}$. In the case of complete or approximately complete distribution (such as in BGS), $F$ is identity function. In my previous work [2], in order to keep the framework of NSM using Tsallis entropy, I proposed $F\left(p_{i}\right)=p_{i}^{q}$ so that

$$
\sum_{i=1}^{w} p_{i}^{q}=1
$$

where $q$ is Tsallis entropy index. Since $p_{i}<1$, we have to set $0<q<\infty$. $q=0$ should be avoided because it leads to $p_{i}=0$ for all states. $p_{i}^{q}$ can be called effective probability which allows relating the parameter $q$ to observed results and so to interactions. According to Eq.(3), Eq.(2) should be written as

$$
\bar{O}=\sum_{i=1}^{v} p_{i}^{q} O_{i}
$$

This incomplete normalization is possible whenever the phase space is partially known or accessible. It is well known that the systems with complex interactions show often fractal or chaotic phase space, in which a complete calculation of probability becomes in general impossible because the space is not integrable or differentiable due to, among others, singular points. In this sense, a possible justification of Eq.(3) can be noticed in [7] discussing nonadditive systems and probability distributions on fractal and multifractal supports, although at that stage the work was not connected to anomalous normalization such as Eq.(3). Considering some simple self-similar fractal structures (e.g. Cantor set), one can obtain :

$$
\sum_{i=1}^{w k}\left[\frac{V_{i}(k)}{V(0)}\right]^{\frac{d_{f}}{d}}=1
$$

where $V_{i}(k)$ may be seen as the segments of the fractal structure at a given iteration of order $k, V(0)$ a characteristic volume of the fractal structure embedded in a $d$-dimension Euclidean space, $d_{f}=\ln n / \ln m$ 
is the fractal dimension, $n$ the number of segments replacing a segment of the precedent iteration, $m$ the scale factor of the iterations and $w_{k}=n^{k}$ the total number of segments at the $k^{\text {th }}$ iteration. If we suppose that the fractal structure is a $d_{f}$-dimension phase space containing homogeneously distributed points, the complete probability distribution at the $k^{\text {th }}$ iteration should be defined as $p_{i}=\frac{V_{i}(k)}{V_{k}}=V_{i}(k) / \sum_{i=1}^{w_{k}} V_{i}(k)$ where $V_{k}$ is the total volume occupied by the state points at the $k^{\text {th }}$ iteration. This distribution obviously sums to one. The problem is that $V_{k}$ is an indefinite quantity which may diverges or shrinks to zero as $k$ increases and, strictly speaking, can not be used to define probability definition. In addition, $V_{k}$ is not differentiable (or integrable) and contains inaccessible singular points. Thus the probability defined above cannot be exactly computed. Now if we define $p_{i}=\frac{V_{i}(k)}{V_{0}}$ as a physical or effective distribution, then we have $\sum_{i=1}^{w_{k}}\left[V_{0} / V(0)\right]^{d f / d} \quad p_{i}^{d} f / d=1$ where $V_{0}$ is a completely accessible and infinitely differentiable support on which the calculation of $p_{i}$ is possible. If we choose $V_{0}=V(0)$, i.e. a $d$-dimension volume containing the $d_{f}$-dimension fractal volume, we get Eq.(3) with $q=d_{f} / d$. The conventional normalization Eq.(1) can be recovered when $d_{f}=d$.

The above example is only a case of distribution on simple fractal structure. It has been shown [8] that, in more complicated cases, e.g., on multifractals composed of overlapping sub-fractals, Eq.(3) always holds if the total joint probability is given by the product of the probability distributions of the sub-fractals.

As mentioned above, Eq.(3) and (4) have been applied to NSM established with Tsallis entropy $S=-k\left(\sum_{i=1}^{w} p_{i}-\sum_{i=1}^{w} p_{i}^{q}\right) / 1-q$. In fact, Tsallis entropy implies an information measure in the form $\ln _{q}\left(1 / p_{i}\right)=\frac{\left(1 / p_{i}\right)^{q-1}-1}{q-1}$, a nonadditive generalization of Hartley formula $\ln \left(1 / p_{i}\right)[2]$. In this case, the normalization of $p_{i}^{q}$ is necessary if we want the entropy to have same nonextensive properties and variance as generalized Hartley information. Of course, different form of the function $F$ will lead to different statistics. For example, if we use Hartley formula as information measure and define expectation value for additive entropy and energy with $F(x)$ satisfying $\frac{\partial F(x)}{\partial x}=\frac{1 / x}{\alpha-\ln x-(\ln Z x)^{1 / \gamma}}$ where $\gamma$ is an empirical parameter, maximum entropy will lead to the stretched exponential distribution [9] $p_{i}=\frac{1}{Z} e^{-\left(\beta e_{i}\right)^{\gamma}}$ where $e_{i}$ is the positive energy of the system at state $i, \alpha$ and $\beta$ are the Lagrange multipliers related respectively to the normalization and energy constraint $U=\sum_{i=1}^{w} F\left(p_{i}\right) e_{i}$. When $\gamma=1$, we can recover $\frac{\partial F(x)}{\partial x}=\frac{1}{x}$ just as in BGS. 
In general, a system becomes nonextensive if it contains interacting parts. But in what follows, we will apply incomplete normalization to extensive systems. This attempt is partially inspired by the insufficiency of the nonextensive quantum distributions of NSM to describe correlated electrons in weakly coupling regime, as discussed in reference [5].

\section{Extensive Incomplete Statistics (EIS)}

Now we suppose that the system of interest has $N$ interacting elements and that the information $I(N)$ needed to specify all the elements, as well as the physical quantities, are additive (e.g. $H=\sum_{j} H_{j}$ where $H$ is the Hamiltonian of the compound system and $H_{j}$ that of the $j^{\text {th }}$ element). Under these harsh conditions, we can postulate [1] :

1) $I(1)=0$ (no information needed if there is only one element)

2) $I(e)=1$ (information unity)

3) $I(N)<I(N+1)$ (more information needed with more elements)

4) $I\left(\prod_{i=1}^{w} N_{i}\right)=\sum_{i=1}^{w} I\left(N_{i}\right) \quad$ (additivity of the information needed to specify simultaneously $w$ subsystems containing respectively $N_{i}$ elements)

5) $I(N)=I_{w}+\sum_{i=1}^{w} p_{i}^{q} I\left(N_{i}\right)$ (additivity of information measure in two steps, where $p_{i}=N i / N$ is the probability to find an element in the $i^{\text {th }}$ subsystem and $I_{w}$ the information needed to determine in which subsystem the element would be found. )

Only the postulate 5 is different from the conventional form because $p_{i}$ is replaced by $p_{i}^{q}$ due to IPD.

As usual, the postulates 1-4 yield Hartley formula $I(N)=\ln N$. The postulate 5 becomes $\ln N=I_{w}+\sum_{i=1}^{w} p_{i}^{q} \ln N_{i}$ which can be recast into

$$
I_{w}=\sum_{i=1}^{w} p_{i}^{q} \ln \left(1 / p_{i}\right)
$$

Now we define an entropy as follows :

$$
S=k \sum_{i=1}^{w} p_{i}^{q} \ln \left(1 / p_{i}\right)
$$

which obviously becomes Gibbs-Shannon entropy $S_{G S}$ when $q=1$. This limit identifies $k$ to Boltzmann constant. It is straightforward to verify that $S$ has the same properties as $S_{G S}$.

For microcanonical ensemble, we have $S=(k / q) \ln w$ which decreases with increasing $q$ value. In general, $\Delta S=S-S_{G S}<0$ (or $>0$ ) if $q>1$ (or $q<0$ ) as shown in Fig.(1). This result is consistent with the fact that the entropy of a lattice of chaotic maps increases with strong coupling [9]. The reader will find later that decreasing $q$ implies increasing coupling for some correlated systems. 


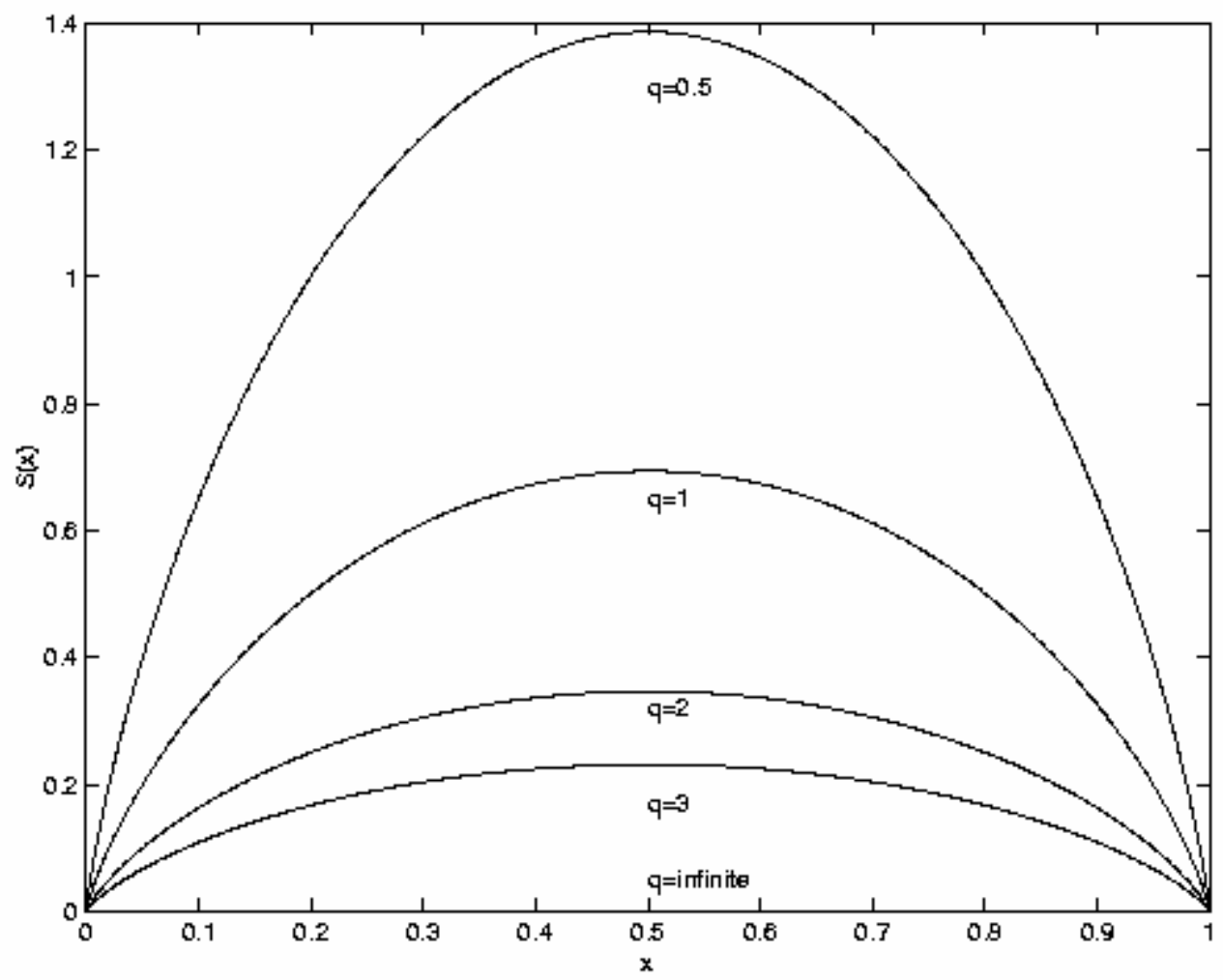

Figure 1. $q$-dependence of the concavity of the extensive generalized entropy $S$ with a two probability distribution $p_{1}=x$ and $p_{2}=1-x$.

For canonical ensemble, the maximum entropy subject to the constraints from Eq.(3) and (4) leads to :

$$
p_{i}=\frac{1}{Z} e^{-\beta e_{i}}
$$

with $Z=\sum_{i=1}^{w} e^{-q \beta e_{i}}$. The Lagrange multiplier $\beta$ can be determined by $k \beta=\frac{1}{T}=\frac{\partial S}{\partial U}$ where $T$ is the absolute temperature and $U$ the internal energy of the system given by $U=\sum_{i=1}^{w} p_{i}^{q} e_{i}$. It is easy to verify that $U=\frac{\partial}{\partial \beta} \ln Z$.

For grand canonical ensemble, the average number of particles is calculated by $U=\sum_{i=1}^{w} p_{i}^{q} N_{i}$ which can be used as a constraint of maximum entropy. The partition function is then given by $Z=\sum_{i=1}^{w} e^{-q \beta\left(e_{i}-\mu N_{i}\right)}$ which gives 


$$
\bar{N}=\frac{1}{\beta} \frac{\partial}{\partial \mu} \ln Z
$$

For quantum particle systems, the usual procedures using Eq.(9), $e_{i}=\sum_{k}\left(n_{k}\right)_{i} \varepsilon_{k}$ and $N_{i}=\sum_{k}\left(n_{k}\right)_{i}$ will lead to

$$
\bar{n}_{k}=\frac{1}{e^{q \beta\left(\varepsilon_{k}-\mu\right)} \pm 1}
$$

where $\bar{n}_{k}$ is the average occupation number of the one-particle state $k$ of energy $\varepsilon_{k}$ and $\mu$ the chemical potential. "+" is for fermions and "-" for bosons.

\section{Examples of Incompleteness Effect}

Now I am presenting examples of the applications of EIS to some simple models. The reader should regard them as demonstrations of the incompleteness behaviors of physical systems.

\section{"Ideal Gas" Model}

The usual calculations give :

$$
\begin{gathered}
Z=\left[\frac{V}{h^{3}}\left(\frac{2 \pi m k T}{q}\right)^{\frac{3 N}{2}}\right]^{\frac{1}{q}} \\
U=\frac{3}{2 q} N k T
\end{gathered}
$$

and

$$
C_{v}=\frac{3}{2 q} N k
$$

where $h$ is the Planck constant.

The effect of the incompleteness can be estimated through the energy difference

$$
\Delta U=U-U_{0}=\left(\frac{1}{q}-1\right) \frac{3}{2} N k T
$$

which is positive (implying repulsion type interactions) for $q<1$ and negative (attraction type interactions) for $q>1$ ( $U_{0}$ is the internal energy of the conventional ideal gas when $q=1$ ).

The reader may be surprised by the treatment of ideal gas with a theory for systems including nonextensive interactions. From the viewpoint of IS, this is just a good example of the philosophy of generalized theory with empirical parameter $q$ which is introduced to "absorb" the effects of "unaccessible" interactions or their effects. In this way, interacting systems may be mathematically treated as noninteracting or conventional ones including only the describable interactions. So we are 
entitled to use, e.g., $\frac{p^{2}}{2 m}$ for the total energy of interacting "free particle", where $p$ is the momentum and $m$ the mass of the particle. The extra interaction energy is "absorbed" in the parameter $q$ when it has different values from unity.

\section{Transport Phenomena of Ideal Gas}

Let $W$ represent the number of particle (of mass $m$ ) collisions happening per second per unit volume, a usual calculation [11] will give :

$$
W=2 n^{2} \sigma \sqrt{\frac{k T}{q \pi m}}=n^{2} \sigma \bar{v} \sqrt{\frac{2}{\pi}}
$$

where $n$ is the particle density and $\bar{v}=\sqrt{\frac{2 k T}{q m}}$ is the most probable speed of a particle. Let the mean free path of a particle be denoted by $\lambda$ the collision time $\tau$ (duration of $\lambda$ ) is defined as follows :

$$
\tau=\frac{\lambda}{\bar{v}}=\frac{n}{2 W}=\frac{1}{4 n \sigma} \sqrt{\frac{q \pi m}{k T}}
$$

In this framework, $\lambda$ does not change with respect to BGS case, but $\tau$ is linked to $q$ and increases with increasing $q$ value. This behaviour of $\tau$ can affect the electrical conductivity $\sigma_{e}$ of metals with free electron model because

$$
\sigma_{e}=\frac{n e^{2} \tau}{m}=\frac{e^{2}}{4 \sigma} \sqrt{\frac{q \pi}{m k T}}
$$

which increases with increasing $q$ value.

\section{Blackbody}

The generalized Planck law is given by

$$
\rho_{q}(v, T)=\frac{8 \pi h v^{3}}{c^{3}} \frac{1}{e^{q \beta h v}-1}
$$

where $v$ is the emission frequency and $c$ the light speed. The generalized Stephan-Boltzmann law is given by $E_{q}(T)=\frac{\sigma}{q} T^{4}$ where $\sigma$ is the usual Stephan-Boltzmann constant. The distribution Eq.(18) is plotted in Figure 2 for different $q$ values to show the effect of the incompleteness of information. We note that this EIS generalized blackbody is essentially different from the nonextensive one [12]. Firstly, the high energy cutoff of nonextensive blackbody is absent in Eq.(18). Secondly, the Einstein emission and absorption coefficients can be easily shown to satisfy $B_{21}=B_{12}$ in this work as in the case of the 


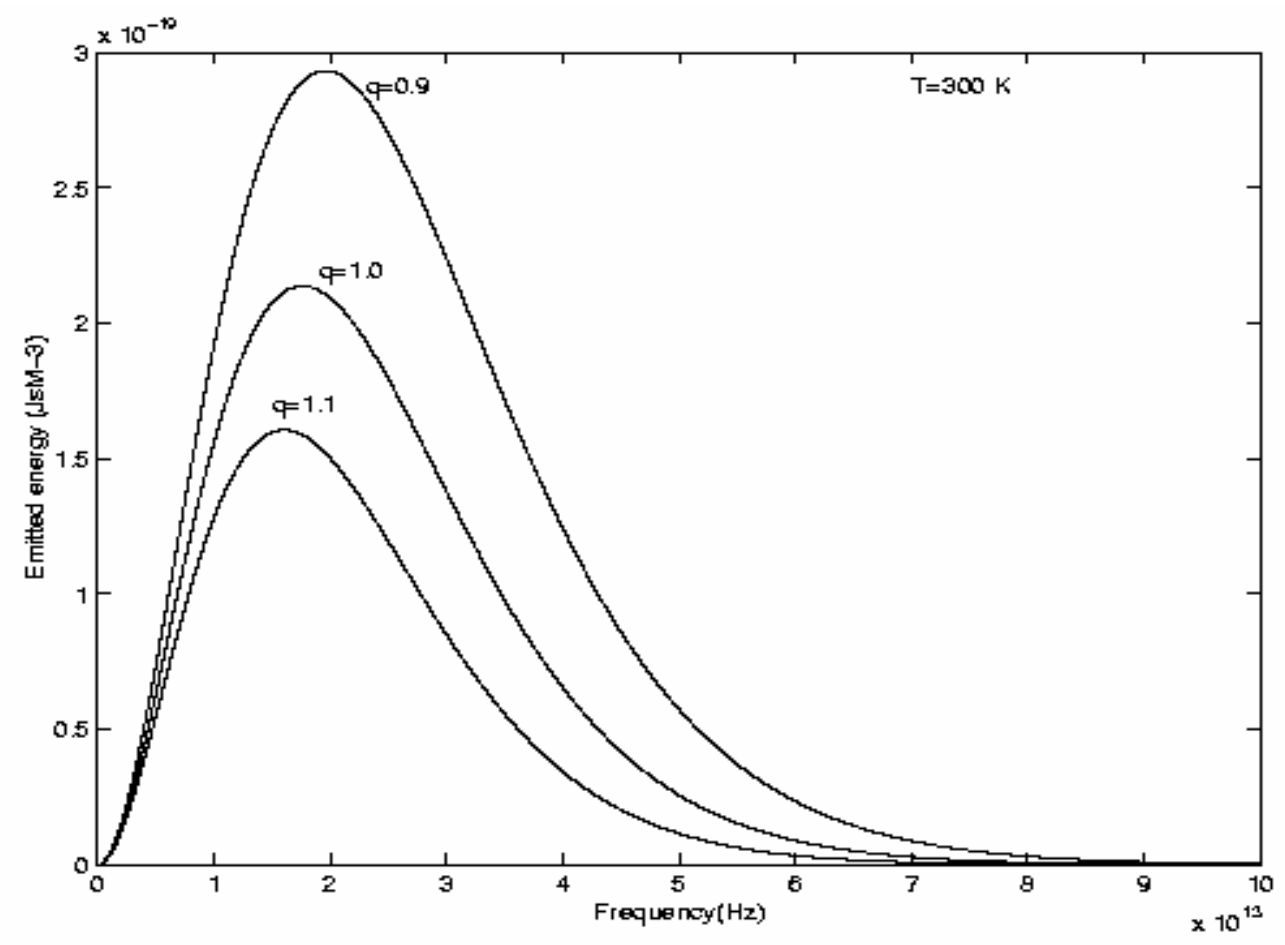

Figure 2. Extensive generalized distribution of blackbody. It can be seen that the total emitted energy and the maximal frequency of the emission increase when $q$ decreases.

conventional Bose-Einstein theory. But with nonextensive blackbody, the ratio $B_{21} / B_{12}$ varies from zero to infinity according to $q$ value [13] which possibly opens a door for the interpretation of the behaviour of the micro chaotic laser [14] which still remains a mystery.

\section{Ideal Fermion Gas}

From the EIS fermion distribution given by Eq.(10), it is easy to verify that the Fermi energy $\varepsilon_{F}^{0}$ at $T=0$ in the generalized version is the same as in the conventional Fermi-Dirac theory. The zero temperature limit of the distribution is therefore not changed.

If $q$ is not very different from unity and $T$ is not too high, the derivative of $\bar{n}$ does not vanish only when $\varepsilon \approx \varepsilon_{F}$. In this case, we can use Sommerfeld integral [15] to calculate Fermi energy $\varepsilon_{F}$ and internal energy for $3 D$ fermion gas. The result is :

$$
\varepsilon_{F} \cong \varepsilon_{F}^{0}\left[1-\frac{\pi^{2}}{12}\left(\frac{k T}{q \varepsilon_{F}^{0}}\right)^{2}\right]
$$

and 


$$
U \cong U_{0}\left[1+\frac{5 \pi^{2}}{12}\left(\frac{k T}{q \varepsilon_{F}^{0}}\right)^{2}\right]
$$

where $U_{0}=\frac{3}{5} N \varepsilon_{F}^{0}$ is the internal energy of fermion gas at $T=0$. The above equations show that the decrease of $q$ leads to internal energy increase and a drop of Fermi level. This is because that, when $q<1$, the particles are driven by the repulsion from the lower energy states to higher ones. We can see from Eq.(10) that, If $q=0$ with $T>0$, the repulsion would be so strong that all states are equally occupied. On the contrary, if $q \rightarrow \infty$, all particles are constrained by the attraction to stay at the lowest states, like the case of zero temperature distribution. This $q$ effect can be seen in Figure 3 where Eq.(10) is plotted for 1- $D$ fermion gas with different $q$ values smaller than unity.

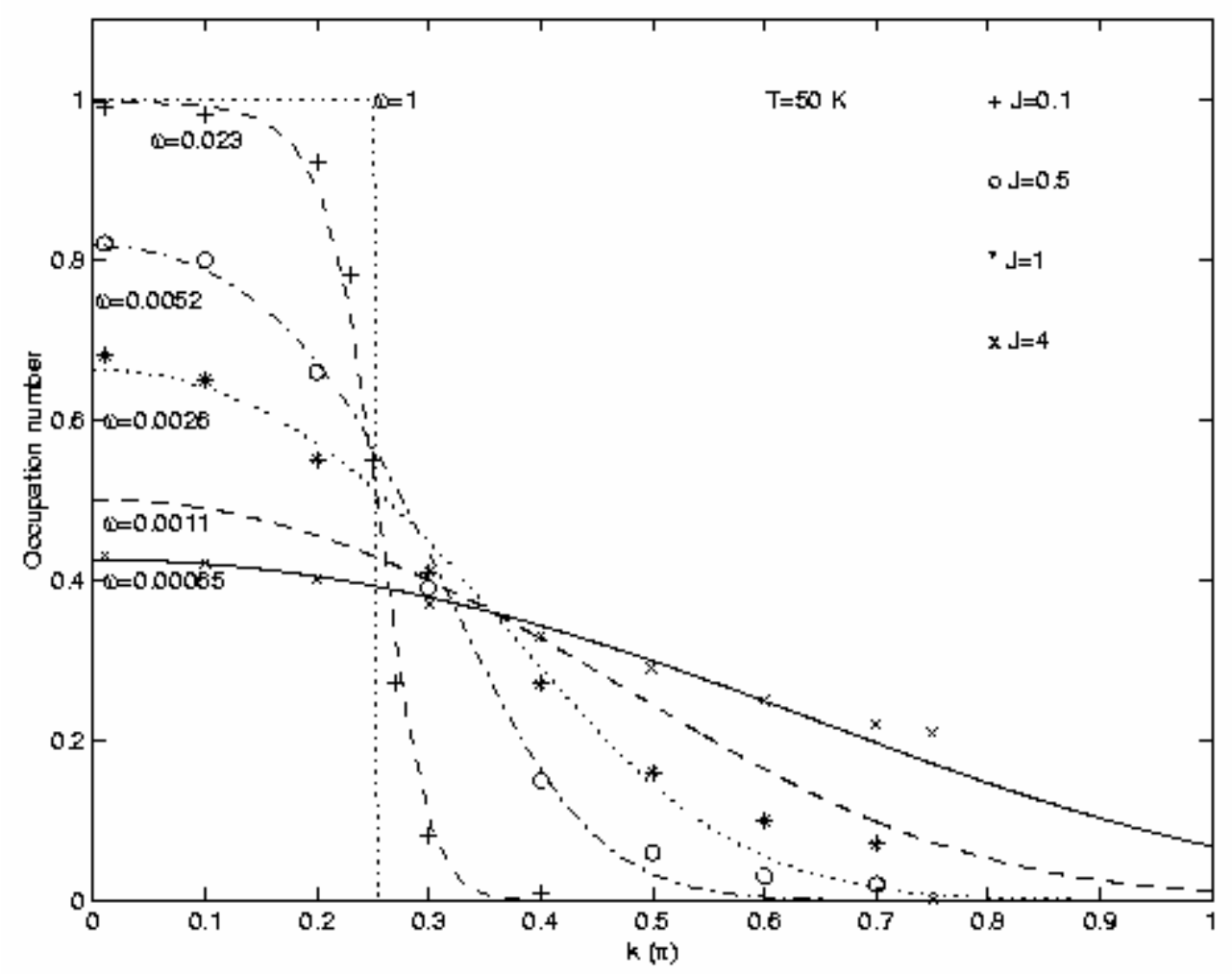

Figure 3. Comparison of IS fermion distribution (lines) at various values of $q$ with the numerical results (symbols) of Moukouri el al on the basis of Kondo lattice $t-J$ model for different coupling constant $J$ [15]. We note the flattening of $n$-drop at Fermi energy $\varepsilon_{F}$ with decreasing $q$ value. When $q=0$, we have $n=1 / 2$ for all possible states. When $q \rightarrow \infty$, we have $n=1$ for all states below $\varepsilon_{F}$ and $n=0$ for all states above $\varepsilon_{F}$ for any temperature. In the calculations, the density of electrons is chosen to give $k_{f}^{0}=0.35 \pi$ in the first Brillouin zone. We see that the lines fit well the numerical results for about $J<2$. When the coupling is stronger, a long tail in the KLM distributions begins to develop at high energy and cannot be reproduced by IS distribution. A new Fermi surface starts to appear and a 
sharp $n$ drop (cutoff) takes place at about $k=0.7 \pi$. These strong correlation effects are absent in the present IS fermion distribution.

The heat capacity $\left(C_{V}\right)_{q}$ and the magnetic susceptibility $\chi_{q}$ of electron gas can be given by:

$$
\left(C_{V}\right)_{q} \cong U_{q}^{0} \frac{5 \pi^{2}}{12}\left(\frac{k}{q \varepsilon_{F}^{0}}\right)^{2} T=\frac{\gamma_{0}}{q^{2}} T
$$

and

$$
\chi_{q} \cong \chi_{0}\left[1-\frac{\pi^{2}}{12}\left(\frac{k T}{q \varepsilon_{F}^{0}}\right)^{2}\right]
$$

where $\gamma_{0}$ is the conventional coefficient of the electronic heat capacity and $\chi_{0}$ the conventional susceptibility of electron gas at $T=0$.

With the help of Eq.(21), the ratio of the effective mass $m_{t h}$ to the mass $m$ of an electron can be related to the parameter $q$ as follows :

$$
\frac{m_{t h}}{m}=\frac{\left(C_{V}\right)_{q}(\text { observed })}{C_{V}(\text { theoretical })}=\frac{1}{q^{2}}
$$

For heavy fermions, we surely have $q<1$. The reader will find below that this fact is confirmed by the $q$ values found for correlated electrons. We remember that above relationships are valid only for $q$ and $T$ values which keep the sharp $n$ drop at $\varepsilon F$.

On the other hand, in Figure 3, the calculations are valid for any $q$ value. For small $q$, we note a flattening of the $n$-drop at $\varepsilon_{F}$ with decreasing $q$ values. This is just a behaviour of correlated electrons observed in some experimental and numerical results [16-21]. Figure 3 shows a comparison of the momentum distribution (lines) given by Eq.(10) for 1- $D$ fermions with numerical results (symbols) given by numerical simulation based on Kondo lattice $t$ - $J$ model (KLM) [17]. We see that IS momentum distribution fits well the numerical results for about $J<2$, the weak coupling regime [17]. When the coupling is stronger $(J>2)$, a long tail in the KLM distributions begins to develop at high energy and can not be fitted with present fermion distribution. In addition, in the strong coupling regime, a new Fermi surface starts to appear and a sharp $n$ drop (cutoff) takes place at about $k=0.7 \pi$. These strong correlation effects are absent in the present EIS fermion distribution. Similar result is obtained with other numerical results [17,22]. This is consistent with the fact that high energy cutoff does not exist in EIS. On the other hand, the sharp $n$ drop at a higher $\varepsilon_{F}$ than the conventional one is indeed observed in NIS fermion distribution [5]. This result confirms our conjecture that EIS is only valid for weak interaction cases due to its extensive nature and that nonextensive effect should be considered whenever interactions become stronger. 


\section{Conclusion}

From above results, we note that the introduction of parameter $q$ is equivalent to replacing $k$ in the conventional BGS theory by $k_{q}=k / q$. One may say that the incomplete q-normalization turns out to be a renormalization of the constant without any change of physics. It should be noticed that the value of Boltzmann constant has been determined for noninteracting systems. So any modification of this value undoubtedly implies physical changes in the studied system as well as in the corresponding theory. The modification given by EIS is a simple one as we guessed considering its validity for the cases where nonextensive effect of interactions can be neglected. The successful fitting of the heavy fermion distributions shows that this modification reflects at least a part of the reality and may be useful.

Summing up, the conventional BGS statistical mechanics is generalized for extensive systems on the basis of the idea that we sometimes can not treat all the information about complex systems so that the physical or observable probabilities become incomplete and do not sum to one. A generalized additive entropy is obtained by using a so called incomplete normalization with a empirical parameter

$q$ which is intended to "absorb" the effect of complex correlations or interaction and can be related to the energy of the studied system. So, in general, $q<1$ and $q>1$ imply respectively repulsive and attractive effect of the complex correlations. This extensive incomplete statistics is shown to be able to reproduce very well the quantum distributions of correlated heavy electrons in weak coupling regime. On the other hand, EIS fails to describe strongly correlated conduction electrons and localized $f$ electrons for which nonextensive effect should be taken into account.

\section{Acknowledgments}

I acknowledge with great pleasure the useful discussions with Professors A. Le Méhauté, Dr. L. Nivanen and Prof. J.P. Badiali on some points of this work.

\section{References}

1. A. Rényi. Calcul de probabilité. Paris, Dunod, 1966, 522.

2. Q. A. Wang. Chaos, Solitons \& Fractals 2001, 12, 1431, for erratum, see cond-mat/0009343.

3. Q. A. Wang. Nonextensive statistics and incomplete information. Euro. Phys. J. B 2002, 26, 357.

4. Q. A. Wang. Quantum gas distributions prescribed by factorization hypothesis of probability. Chaos, Solitons \& Fractals 2002, 14, 765.

5. Q. A. Wang and A. Le Méhauté. Extensive form of equilibrium nonextensive statistics. $J$. Math. Phys 2002, 43, 5079-5089.

6. C. Tsallis. Chaos, Solitons \& Fractals 1995, 6, 539; C. Tsallis, J. Statis. Phys. 1988, 52, 479; EMF. Curado and C. Tsallis. J. Phys. A:Math.Gen. 1991, 24, L69; C. Tsallis, R. S. Mendes and A. R. Plastino, Physica A 1999, 261, 534; Silvio R. A. Salinas and C. Tsallis. Brazilian Journal of Physics (special issue: Nonextensive Statistical Mechanics and Thermodynamics), 1999, 29.

7. C. Tsallis. Fractals 1995, 3, 541; D.H. Zanette. Physica A 1996, 223, 87-98. 
8. Q. A. Wang, L. Nivanen, A. Le Méhauté, and M. Pezeril. A treatment of stationary nonextensive systems with different $q$ indices, cond-mat/0305398

9. P. Jund, R. Julien and I. Campbell. Phys. Rev. E 2001, 63, 036131.

10. A. M. Batista and R. L. Viana. Physica A 2002, 308, 125-134.

11. K. Huang. Statistical Physics; Wiley, New York, 1987, Chap.5, 2nd ed.

12. Q. A. Wang, and A. Le Méhauté. Phys. Lett. A 1997, 237, 28; Q. A. Wang, L. Nivanen and A. Le Méhauté. Physica A 1998, 260, 490-498.

13. Q. A. Wang, and A. Le Méhauté. Phys. Lett. A 1998, 242, 301-306.

14. C. Gmachl, F. Capasso, E. E. Narimanov, J. U. Nokel, A. D. Stone, J. Faist, D. L. Sivco, A. Y. Cho. Science 1998, 280, 1493-1656.

15. Donald A. McQuarrie. Statistical Mechanics; New York, Harper and Row, 1973, 167.

16. R. Eder, O. Rogojanu and G.A. Sawatzky. Many-body band structure and Fermi surface of the Kondo lattice, cond-mat/9711248.

17. S. Moukuri and L. G. Caron. Phys. Rev. B 1995, 252, R15723; S. Moukuri and L. G. Caron. Fermi surface of the one-dimensional Kondo Lattice Model, cond-mat/9607123.

18. C. Grober and R. Eder. Fermiology of a $1 D$ heavy electron model, cond-mat/9812345.

19. F. Ronning, C. Kim, D. L. Feng, D. S. Marshall, A. G. Loeser, L. L. Miller, J. N. Eckstein, I. Bozovic, Z. X. Shen. Photoemission evidence for a Remnant Fermi surface and d-wave-like dispersion in insulting $\mathrm{Ca}_{2} \mathrm{CuO}_{2} \mathrm{Cl}_{2}$. Science 1998, 282, 2067.

20. W. O. Putikka, M. U. Luchini and R. R. P. Singh. Violation of Luttinger's theorem in the twodimensional t-J model, cond-mat/9803140.

21. W. O. Putikka, M. U. Luchini and R. R. P. Singh. Does the two-dimensional t-J model have hole pockets? cond-mat/9803141.

22. Q. A. Wang. Correlated electrons and generalized statistics. Euro. Phys. J. B 2003, 31, 75-79.

\section{Note}

${ }^{1}$ In 1654, the Chevalier de Méré, a gamester, proposed to Blaise Pascal the following problem : two players of equal skill want to leave the table before finishing their game. Their scores and the number of points which constitute the game being given, it is desired to find in what proportion they should divide the stakes. In his answer to this question, Pascal laid down the principles of the theory of probabilities.

(C) 2003 by MDPI (http://www.mdpi.org). Reproduction for noncommercial purposes permitted. 